\title{
UTILISATION DE LA FORMULE D'EINSTEIN. COMPARAISON AVEC LA FORMULE DE MEYER-PETER DANS LE DOMAINE DU DÉBUT D'ENTRAINEMENT.
}

par M. Alquier *

\section{Introduction}

Devant l'incompatibilité qui vient d'être soulignée, nous avons pensé regarder les résultats que pourrait donner une utilisation d'une formule du style d'Einstein, comparée à celle de Meyer-Peter. En effet, force nous est de constater que dans l'expérience de la Bruche, les charriages totaux enregistrés restent très faibles et que par suite, la force tractrice et la force tractrice critique ont toujours été voisines l'une de l'autre. Or dans les formulations d'Einstein, à ces notions sont associées des probabilités qui peuvent vraisemblablement permettre d'analyser plus finement ce qui se passe au début de charriage. Citons à cet effet Frijlink [1]:

«Dans la formule empirique de Meyer-Peter et Muller, il est conçu une valeur critique de la tension de frottement. Dans les formules de Kalinske et d'Einstein, par contre, qui sont basées sur les théories modernes de la turbulence, on ne rencontre pas une telle valeur. Celle-ci est remplacée par la «probabilité de mouvement» qui diminue toujours à mesure que la tension de frottement devient plus petite.

Ceci correspond à ce que l'on peut voir au commencement du transport. En effet, il y a toujours quelques grains qui sont en mouvement par suite d'un petit coup de turbulence et un repos absolu du matériau de fond n'est presque jamais atteint.

Nous sommes d'avis qu'il est nécessaire que dans les formules de transport, la préférence soit donnée à la probabilité de mouvement, qui concorde plus avec la réalité. C'est surtout pour de très petits transports que l'on obtient alors des résultats plus exacts qu'avec l'emploi d'une valeur limite. 》

Pour simplifier les raisonnements et applications qui vont suivre, nous prendrons en compte uniquement la courbe 2 du graphique 1, figure 1, comme représentative de la courbe:

$$
\mathbf{R}_{\mathrm{II}}=f\left(q_{7}\right)
$$

Par ailleurs, nous admettrons, pour la Bruche, les données suivantes:

granulométrie :

$$
\begin{aligned}
d_{90} & =13,4 \cdot 10^{-2} \mathrm{~m} \\
d_{35} & =8,8 \cdot 10^{-2} \mathrm{~m}
\end{aligned}
$$

largeur affectée pour le transport: $l=15 \mathrm{~m}$

pente : $\quad I=710^{-3}$

\section{Formules de Meyer-Peter}

Influence du choix de la force tractrice critique.

Devant la difficulté précédemment soulignée d'évaluer avec précision la force tractrice critique $\tau_{c}$, nous avons considéré celle-ci comme variable.

Sur la figure 3 apparaît alors :

$1^{\circ}$ Le réseau des courbes selon Meyer-Peter, du débit solide instantané pour:

$$
a=\frac{q_{a}}{q_{l}} \times\left(\frac{k_{s}}{k_{r}}\right)^{3 / 2}=1
$$

et pour trois valeurs:

$$
\begin{aligned}
& \tau_{c}=40 \mathrm{~N} / \mathrm{m}^{2} \text { conduisant à̀ } q_{l}=12 \mathrm{~m}^{3} / \mathrm{s} \\
& \tau_{c}=50 \mathrm{~N} / \mathrm{m}^{2} \text { conduisant à̀ } q_{l}=20 \mathrm{~m}^{3} / \mathrm{s} \\
& \tau_{r}=60 \mathrm{~N} / \mathrm{m}^{2} \text { conduisant à̀ } q_{l}=30 \mathrm{~m}^{3} / \mathrm{s}
\end{aligned}
$$

2" La courbe de Meyer-Peter pour $a=0,715$ : et :

$$
\tau_{c}=50 \mathrm{~N} / \mathrm{m}^{2} \text { conduisant à } q_{l}=45 \mathrm{~m}^{3} / \mathrm{s}
$$

\section{Discussion des résultats.}

Le diagramme précédent met bien en évidence, s'il en était encore besoin, l'erreur énorme que peut entraîner une erreur sur $\tau_{c}$ dans la phase de début d'entrainement.

Ainsi pour un débit liquide de $22 \mathrm{~m}^{3} / \mathrm{s}$, on trouve :

$$
\begin{aligned}
& q \text { solide } \mathrm{kg} / \mathrm{s}=0,5 \text { pour } \tau_{c}=50 \\
& q \text { solide } \mathrm{kg} / \mathrm{s}=22 \text { pour } \tau_{c}=40
\end{aligned}
$$

De la même manière, le choix de $« a »$ influe énormément sur le résultat.

\section{Formules d'Einstein}

\section{$q_{s}=f\left(q_{1}\right)$ selon Einstein.}

Rappelons très brièvement que Einstein bâtit une théorie de charriage conduisant à l'expression d'une probabilité de progression des grains, connue sous le nom de «fonction de charriage d'Einstein $»$, dans laquelle intervient deux fonctions caractéristiques du transport:

\footnotetext{
* Maître-Assistant à l'Institut de Mécanique des Fluides de Toulouse.

*: Professeur à l'Institut de Mécanique des Fluides de Toulouse.

* Section d'Application des Radioéléments de Saclay, Centre d'Etudes Nucléaires.
} 
L'intensité de transport:

$$
\Phi=\frac{q_{s}}{\rho_{s} g}\left(\frac{\rho}{\rho_{s}-\rho}\right)^{1 / 2}\left(\frac{1}{g d^{3}}\right)^{1 / 2}
$$

L'intensité de mouvement:

$$
\psi=\frac{\rho_{3}-\rho}{\rho} \frac{d}{\mathrm{R}_{\mathrm{H}} \mathrm{J}}
$$

Formules dans lesquelles:

$\rho_{s}:$ masse spécifique du sédiment;

$\rho:$ masse spécifique du liquide;

$g$ : accélération de la pesanteur.

Ces deux fonctions étant reliées entre elles par une courbe universelle faisant l'objet de la figure 4 .

Par ailleurs, dans ces formulations, Einstein conseille d'utiliser $d_{35}$ comme calibre représentatif pour les sédiments présentant une gamme "étroite» de granulométrie.

L'application des données sur la Bruche, conduit alors pour les deux fonctions précédentes à :

$$
\begin{gathered}
\Phi=3,5 \cdot 10^{-4} \cdot q_{s} \\
\psi=\frac{21,4}{\mathrm{R}_{\mathrm{II}}}
\end{gathered}
$$

Et par l'intermédiaire de la courbe universelle de la figure 4 au tracé de la courbe de charriage correspondante de la figure 3 .

\section{Comparaison Einstein-Meyer-Peter.}

1) Il est aisé et rassurant de constater que pour les forts débits liquides, entraînant des charriages importants, les deux formulations tendent à donner des chiffres analogues; on comparera utilement les deux courbes de charriage d'Einstein et Meyer-Peter $(a=0,715)$ qui sont parallèles et voisines l'une de l'autre au-delà de $70 \mathrm{~m}^{3} / \mathrm{s}$.

2) Par contre, et conformément à ce qui avait été prévu, leur allure est très différente dans la zone de début de charriage: Einstein prévoit dans cette phase, une mise en mouvement très progressive quasiment insensible aux variations des forces tractrices agissantes, par contre, Meyer-Peter voit une mise en mouvement brutale s'accélérant brutalement avec un accroissement faible du débit liquide, ou au contraire diminuant ou cessant brutalement pour une augmentation locale faible de la force tractrice. Aussi peut-on dire qu'implicitement le premier modèle dans cette phase, nous semble plus près de la réalité.

\section{Charriage total de la Bruche selon Einstein.}

\section{Charriage total de la Bruche.}

Reprenant le processus utilisé lors de l'emploi de la formule Meyer-Peter, on peut pour l'ensemble des trois crues dont on retrouve le tracé sur la figure 1, graphique 2, calculer le charriage total de la Bruche en utilisant une formule d'Einstein, selon :

$$
\mathrm{Q}_{\mathrm{T}}=\int_{0}^{\mathrm{T}} \stackrel{q}{s}(t) d t
$$

Cette opération a été faite graphiquement. On trouve alors :

$$
\mathrm{Q}_{\mathrm{T}}=1200 \mathrm{t}
$$

qu'on rapprochera des $1730 \mathrm{t}$ données par l'expérience de traceurs radioactifs. L'accord apparaît comme très satisfaisant pour une détermination de ce type.

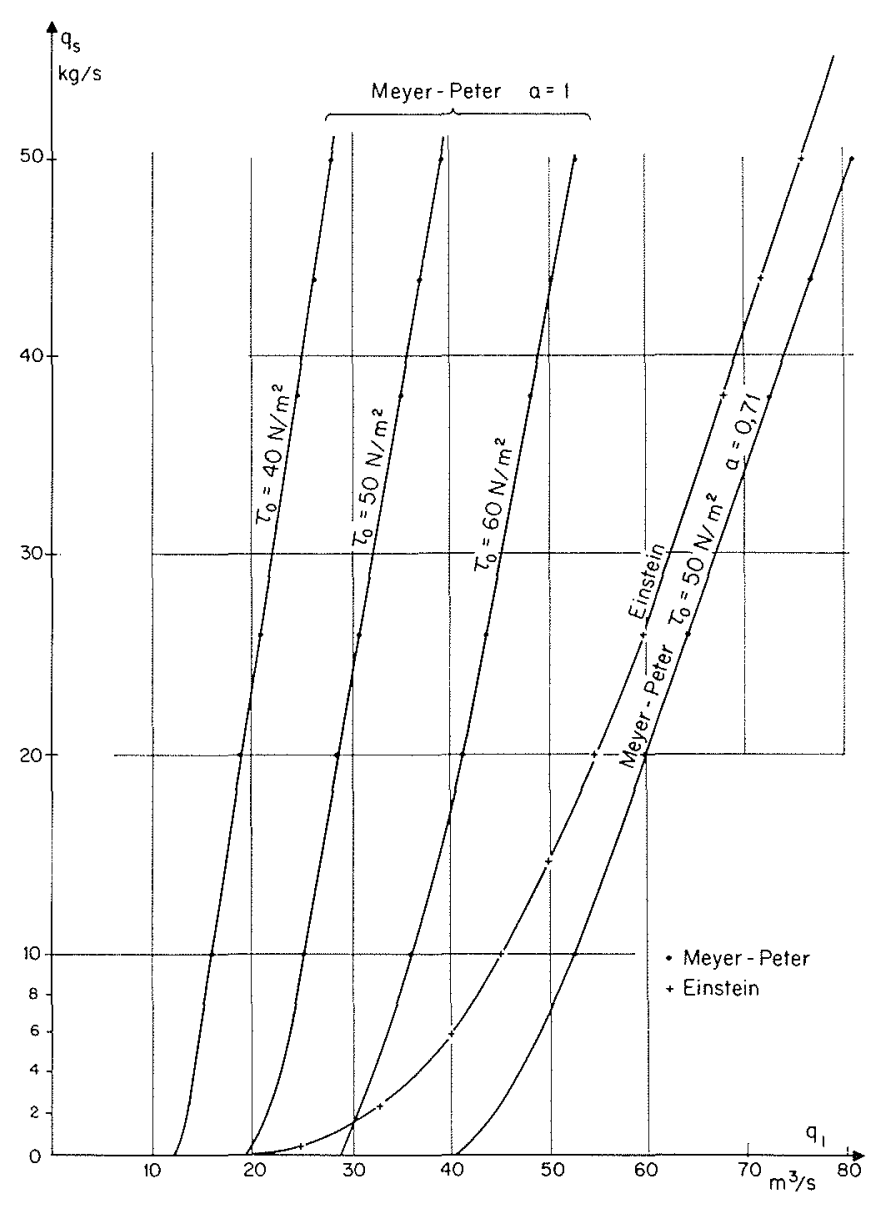

3/ Courbes débit solide - débit liquide.

NotTION DE DÉBUt de CHARRIAGE.

Certes on ne peut dans l'absolu parler de débit critique de début de charriage dans une formule d'Einstein, puisque tout débit liquide si petit soit-il, correspond à un débit de charriage.

Cependant il est intéressant de constater que la courbe de débit de charriage (fig. 3) décolle précisément dans la zone de 15 à $20 \mathrm{~m}^{3} / \mathrm{s}$ que nous avions par ailleurs située comme étant la zone de début de charriage.

Ainsi selon Einstein, à $17,5 \mathrm{~m}^{3} / \mathrm{s}$, la Bruche charierait

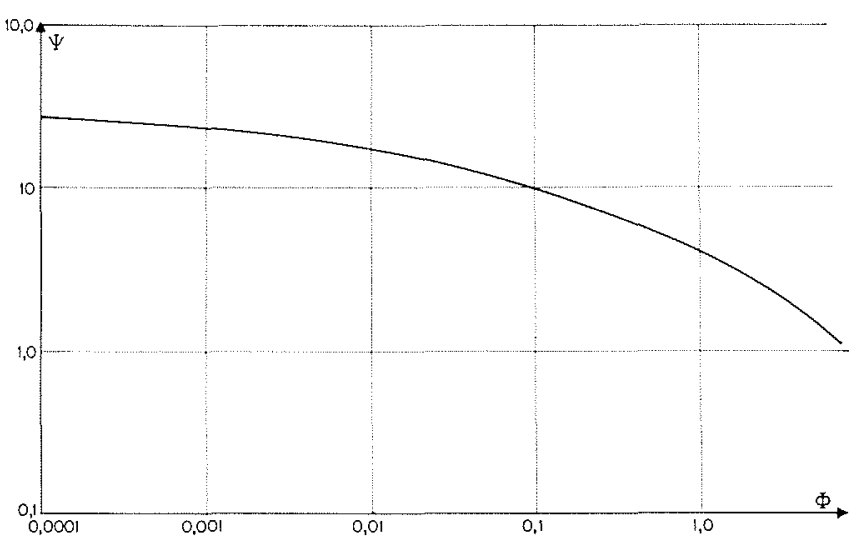

4/ Fonction universelle d'Einstein $\Psi=f(\Phi)$. 


\section{G. COURTOIs et al.}

$0,05 \mathrm{~kg} / \mathrm{m} / \mathrm{s}$ soit $0,75 \mathrm{~kg}$ sur toute la rivière. En prenant $1 \mathrm{~kg}$ pour poids moyen du galet, ceci représente un galet toute les 1 à $2 \mathrm{~s}$ sur toute la largeur. Ceci à notre sens correspond d'une façon satisfaisante, à une notion empirique de début de charriage.

\section{Conclusions}

De l'expérience que nous avons des transports solides dans les torrents de montagnes, on s'aperçoit que fréquemment, il existe deux types de phénomènes:

- Dans les années de grandes crues (crues des dix ans par exemple), les charriages sont très importants et représentent la majorité des apports aux vallées, aux barrages, à la mer. La méthode de traceurs radioactifs s'avère alors relativement inefficace compte tenu de la dispersion des sédiments tant en longueur, qu'en profondeur.

Dans ce domaine des forts charriages, les diverses équations de charriage connues semblent se montrer relativement équivalentes, et peuvent permettre d'atteindre un ordre de grandeur du charriage, à comparer aux méthodes pratiques qui peuvent être mises en place notamment les cubages, par relevés systématiques des profils.

- Par contre dans les années courantes, les charriages restent faibles. Les traceurs s'avèrent alors de bonnes méthodes d'évaluation, et la comparaison des résultats obtenus avec les formules semi-empiriques devrait alors se faire par l'intermédiaire des formules qui donnent priorité aux notions statistiques de force agissante et de transport tels que Einstein, Kalinske, Brown, etc.

On évitera alors l'emploi de formulations faisant apparaître directement ou indirectement les notions de force tractrice et de débit critique de début d'entraînement, telles que les fonctions de Meyer-Peter, Schoklitsch, Duboys, etc.

Bibliographie

[1] Frujkink (H.C.). - Discussion des formules de débit solide de Kalinske, Einstein, Meyer-Peter et Muller: « Transport hydraulique et décantation des matériaux solides " $2^{\text {c Journées }}$ de l'Hydraulique, Grenoble 25-29 juin 1952. Organisateur S.H.F. Publié en 1953 par La Houille Blanche, p. 98-103.
M. le Président: «Je remercie M. Courrors de son exposé extrêmement dense. Je le remercie particulièrement d'avoir bien voulu exposer les principes et un crtain nombre de méthodes, ce qui a permis à des personnes non averties comme moi de suivre son intervention. Je pense qu'il serait bon de vous donner la parole de façon à éclaircir certains points. Y-a-t-il des personnes qui ont des questions à poser à $M$. CourTols? »

M. Olive (Centre de Recherches géodynamiques de Thonon-lesBains) :

«Comment faut-il faire pour extrapoler des résultats que vous avez à une certaine échelle sur l'ensemble du phénomène? Je prends un exemple: pour la Gironde, quand on a fait des expériences de marquage on a des résultats qui s'étendent sur une zone de 1 à $2 \mathrm{~km}$, alors que les phénomènes d'envasement de la Gironde s'étendent sur $100 \mathrm{~km}$. Le même phénomène se passe lorsque vous obtenez des résultats sur quelques heures ou quelques jours ou pendant un mois. Or, le phénomène va être important au moment des crues ou pendant des périodes de temps plus longues. „

M. Courtors : «Effectivement, c'est un point important. Nous étudions un phénomène particulier dans une période de temps relativement courte. Les expériences peuvent être relativement longues. Nous avons mené très couramment des expériences sur un an. Toutes les expériences de galets sont sur un an. Elles peuvent être répétées au bout d'un an. Par exemple, avec M. Malre, de Strasbourg, nous avons fait une expérience dans la Bruche l'année dernière, que nous répétons cette année dans des conditions géomorphologiques un peu différentes. Toutes les expériences que nous avons menées avec le Laboratoire Central dHydraulique de France ont duré en moyenne un an. Il existe quand même un certain nombre de phénomènes qui peuvent être étudiés sur des périodes plus courtes. Je m'explique. Les expériences que nous avons faites avec l'Institut de Géologie du Bassin d'Aquitaine étaient faites pour voir quelle était l'action érosive ou l'action dynamique des marées de fort coefficient. Dans ce cas, nous utilisons un radioélément de courte période. Nous travaillons pendant une dizaine de jours. Nous ensemençons la zone trois ou quatre jours avant le maximum de flux et nous travaillons quelques jours après. Donc, nous encadrons un phénomène. De la même manière, des problèmes sont posés par le Génie Civil au sujet du dragage. Il s'agit de savoir comment se forme un courant de turbidité. Ce courant de turbidité se forme extrêmement rapidement. Il y a là un certain nombre d'applications qui peuvent être mises en cuvre avec des traceurs de courte période, bien que je le répète certaines études peuvent durer jusqu'à un an. Il est bien certain que dans le domaine de la recherche et particulièrement dans le domaine qui vous intéresse ou qui intéresse par exemple M. MARTYN, la méthode de traceurs radioactifs n'est pas la panacée. Elle ne permet pas, elle ne permettra jamais, d'atteindre ce qu'il faut appeler « presque » les âges géologiques, bien quion se place au milieu quaternaire. On ne fera pas d'expériences dont les résultats soient valables sur une centaine d'années ou sur plusieurs milliers d'années, comme pourraient vous le donner des informations issues des minéraux lourds. Nous ne pourront pas non plus vous donner des résultats valables sur 100 ou $150 \mathrm{~km}$. C'est à chacun de voir quelles sont les limites de ces techniques et quels sont les renseignements qu'elles peuvent apporter. » 